\title{
Diffusion-weighted magnetic resonance imaging (MRI) without susceptibility artifacts: single-shot stimulated echo acquisition mode (STEAM) MRI with iterative reconstruction and spatial regularization
}

\author{
Dirk Voit, Oleksandr Kalentev, Jens Frahm^^ \\ Biomedizinische NMR, Max-Planck-Institut für biophysikalische Chemie, Göttingen, Germany \\ Correspondence to: Prof. Dr. Jens Frahm. Biomedizinische NMR, Max-Planck-Institut für biophysikalische Chemie, 37070 Göttingen, Germany. \\ Email: jfrahm@gwdg.de.
}

\begin{abstract}
This work describes a new method for diffusion-weighted (DW) magnetic resonance imaging (MRI) without susceptibility artifacts. The technique combines a DW spin-echo module and a single-shot stimulated echo acquisition mode (STEAM) MRI readout with undersampled radial trajectories and covers a volume by a gapless series of cross-sectional slices. In a first step, optimal coil sensitivities for all slices are obtained from a series of non-DW acquisitions by nonlinear inverse reconstruction with regularization to the image and coil sensitivities of a directly neighboring slice. In a second step, these coil sensitivities are used to compute all series of non-DW and DW images by linear inverse reconstruction with spatial regularization to a neighboring image. Proof-of-principle applications to the brain (51 sections) and prostate (31 sections) of healthy subjects were realized for a protocol with two b-values and 6 gradient directions at $3 \mathrm{~T}$. Including averaging the measuring times for studies of the brain at $1.0 \times 1.0 \times 3.0 \mathrm{~mm}^{3}$ resolution $\left(\mathrm{b}=1,000 \mathrm{~s} \mathrm{~mm}^{-2}\right)$ and prostate at $1.4 \times 1.4 \times 3.0 \mathrm{~mm}^{3}$ resolution $\left(\mathrm{b}=600 \mathrm{~s} \mathrm{~mm}^{-2}\right)$ were $2.5 \mathrm{~min}$ and $4.5 \mathrm{~min}$, respectively. All reconstructions were accomplished online with use of a multi-GPU computer integrated into the MRI system. The resulting non-DW images, mean DW images averaged across directions and maps of the apparent diffusion coefficient confirm the absence of geometric distortions or false signal alterations and demonstrate diagnostic image quality. The novel method for DW STEAM MRI of a volume without susceptibility artifacts warrants extended clinical trials.
\end{abstract}

Keywords: Diffusion-weighted magnetic resonance imaging (diffusion-weighted MRI); radial undersampling; inverse reconstruction; spatial regularization

Submitted Jul 31, 2020. Accepted for publication Sep 22, 2020.

doi: 10.21037/qims-20-871

View this article at: http://dx.doi.org/10.21037/qims-20-871

\section{Background}

Diffusion-weighted (DW) magnetic resonance imaging (MRI) is of high clinical relevance because diffusion characteristics differ between normal and pathologic tissue and thus result in diagnostic contrast. In a technical sense, diffusion encoding is commonly accomplished by a DW spin-echo sequence which encompasses a self-compensating set of magnetic field gradients. Because strong gradients need to ensure sufficient signal attenuation of a DW image relative to a non-DW image, DW MRI techniques are very sensitive to (macroscopic) movements. It is therefore

^ ORCID: 0000-0002-8279-884X. 
standard practice to combine a DW spin-echo module with a high-speed (i.e., single-shot) MRI readout which effectively "freezes" the actual condition. In the past, several technical proposals have been made with respect to this strategy.

A preferred solution to overcome the motion sensitivity of DW MRI is the use of an echo-planar imaging (EPI) readout, for recent reviews see $(1,2)$. DW EPI is widely accepted for clinical applications-despite some severe problems such as the pronounced sensitivity to magnetic field inhomogeneity. Unavoidable differences in the magnetic susceptibility of air and tissues frequently lead to geometric distortions as well as false positive or negative signal alterations in DW EPI and compromise the diagnostic accuracy. On the other hand, for clinical applications, DW EPI is currently the method of choice as it offers a signal-to-noise ratio (SNR) and spatial resolution suitable for diagnostic purposes $(1,2)$.

An alternative solution for DW MRI, which avoids the sensitivity to magnetic field inhomogeneity and still allows for high-speed acquisitions, is the use of a single-shot spinecho MRI sequence, for example see $(3,4)$. This technique uses multiple radiofrequency-refocused spin echoes (rather than gradient echoes as for EPI) in order to encode the spatial information. A drawback of this approach, however, is the need for many refocusing pulses with high flip angles which often violates the specific absorption rate (SAR) limit, in particular at high magnetic field strength. As a consequence, such single-shot spin-echo methods are not in general use for human applications.

An even further proposal is a combination of the DW spin-echo sequence with a readout series of stimulated echoes which are generated by radiofrequency pulses with low flip angles and therefore avoid the SAR problem (5). Previous versions for DW single-shot stimulated echo acquisition mode (STEAM) MRI $(6,7)$ solve the susceptibility problem of DW EPI, but suffer from a lower SNR (8). In a recent DW STEAM implementation with undersampled radial trajectories designed for applications to the human brain (9), coil sensitivity maps were obtained from a non-DW dataset by nonlinear inversion (NLINV) with regularization to the coil sensitivity maps of a neighboring cross-section. However, because the actual image reconstructions were accomplished by linear inversion without any spatial regularization, the approach led to only weakly regularized reconstructions with limited numerical stability.

In contrast, the current development relies on a full spatial regularization of the inverse problem which not only refers to the coil sensitivity maps of a directly neighboring section, but more importantly involves the actual image. The resulting procedure offers both low and high spatial frequency information for regularization of the illposed numerical optimization problem. Accordingly, the respective reconstructions of non-DW and DW images are computationally robust and allow for a higher degree of data undersampling and increased acquisition speed. Exploiting the similarity of directly neighboring images formally adopts the same regularization as originally applied for dynamic real-time MRI (10), but now in space rather than in time. A related strategy has recently been shown to rapidly cover a volume by real-time gradient-echo MRI with framewise advancement of the slice position (11). Such reconstructions achieve high spatial fidelity as previously demonstrated for the case of temporal fidelity when using temporal regularization to a preceding image (12). The purpose of this proof-of-concept work is to qualitatively evaluate a spatially regularized DW STEAM MRI method which not only overcomes the EPI susceptibility problem, but also yields sufficient SNR and resolution for future clinical applications.

\section{Methods}

All studies were performed at $3 \mathrm{~T}$ using an MRI system with $80 \mathrm{mT} / \mathrm{m}$ gradients (Magnetom Prisma fit, Siemens Healthineers, Erlangen, Germany). DW single-shot STEAM MRI of the brain and prostate employed a 64-channel head coil or an 18-element thorax coil in conjunction with suitable elements of the spine coil array, respectively. During technical development, subjects without known illness were recruited among the students of the local University. The study was approved by the ethics committee of the Göttingen University Medicine (No. 12/6/15) and written informed consent was taken from all subjects prior to MRI.

A DW single-shot STEAM MRI sequence with pronounced radial undersampling was implemented as described (9). The schematic diagram in Figure 1 illustrates the combination of a DW spin-echo module and a single-shot STEAM MRI readout. A list of experimental parameters is summarized in Table 1. In all cases the method scans a volume in a gapless manner using a series of directly neighboring cross-sections. The actual acquisition is performed in an interleaved multi-slice order to minimize crosstalk in combination with a long repetition 


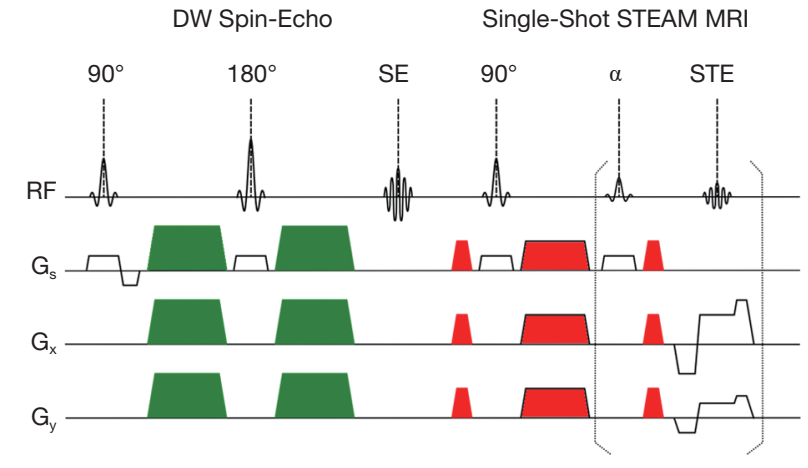

Figure 1 Schematic diagram of a generic diffusion-weighted (DW) stimulated echo acquisition mode (STEAM) magnetic resonance imaging (MRI) sequence combining a DW spin-echo module and a single-shot STEAM MRI sequence with undersampled radial trajectory. The sequence generates a series of stimulated echoes by low-flip angle readout pulses $\alpha$. Green $=$ diffusion-encoding gradients (unipolar version); red = spoiler gradients. $\mathrm{SE}$, spin echo; STE, stimulated echo; RF, radiofrequency pulses; $\mathrm{G}_{\mathrm{s}}$, sliceselection gradient (white); $G_{x}, G_{y}$, radial encoding gradients (white).

time (also needed for a large number of slices). The implementation further ensures that datasets of spatially neighboring sections are recorded with 5 complementary sets of radial spokes as developed for real-time MRI with radial trajectories (10). Fat suppression is achieved using a chemical-shift-selective (CHESS) pulse and gradient module (13) before the acquisition of each dataset.

In a first step, a series of optimal coil sensitivities is obtained from a multi-slice acquisition without diffusion encoding. This task is accomplished by NLINV which jointly estimates each image and its associated coil sensitivities. The process employs spatial regularization of the actual image and coil sensitivities to both the image and coil sensitivities of a directly neighboring cross-section. The numerical problem is solved by the iteratively regularized Gauss-Newton method (14) in close correspondence to the NLINV reconstructions introduced for real-time MRI (10) and rapid volume coverage (11) - for mathematical details see these earlier publications. However, the current application does not reduce the number of receive coil channels with use of a principal component analysis, but takes data from all physical receive coils encompassing the imaging volume.

In a second step, the coil sensitivities of the nonlinear inverse reconstruction without diffusion encoding are taken to calculate all series of non-DW and DW images
Table 1 Diffusion-weighted single-shot stimulated echo acquisition mode (STEAM) MRI of the brain and prostate

\begin{tabular}{|c|c|c|}
\hline Parameter & Brain & Prostate \\
\hline Field-of-view $\left(\mathrm{mm}^{2}\right)$ & $224 \times 224$ & $224 \times 224$ \\
\hline Image matrix size & $224 \times 224$ & $160 \times 160$ \\
\hline In-plane resolution $\left(\mathrm{mm}^{2}\right)$ & $1.0 \times 1.0$ & $1.4 \times 1.4$ \\
\hline Slice thickness $/ \mathrm{mm}$ & 3.0 & 3.0 \\
\hline Number of slices & 51 & 31 \\
\hline Number of spokes & 17 & 17 \\
\hline $\operatorname{TR}(\mathrm{ms})^{\mathrm{a}}$ & 10,000 & 6,000 \\
\hline $\mathrm{TE}(\mathrm{SE})(\mathrm{ms})^{\mathrm{b}}$ & 36.0 & 31.2 \\
\hline TR (STE) $(\mathrm{ms})^{c}$ & 7.36 & 7.36 \\
\hline TE $(\mathrm{STE})(\mathrm{ms})^{\mathrm{d}}$ & 8.76 & 8.76 \\
\hline Diffusion gradient directions & 6 & 6 \\
\hline b-values $\left(s \cdot \mathrm{mm}^{-2}\right)$ & $0,1,000$ & 0,600 \\
\hline Averages per b-value ${ }^{e}$ & 3,2 & 9,6 \\
\hline Acquisition time $(\min : s)^{f}$ & $2: 30$ & $4: 30$ \\
\hline \multicolumn{3}{|c|}{$\begin{array}{l}\text { a } \text {, repetition time; }{ }^{\text {b }} \text {, spin-echo time; }{ }^{c} \text {, STEAM repetition time } \\
\text {, stimulated-echo time; }{ }^{e} \text {, numbers refer to the two b-values } \\
\text { shown in the preceding line; }{ }^{f} \text {, total measurement time for multi } \\
\text { slice DW MRI. }\end{array}$} \\
\hline
\end{tabular}

as solutions to a linear inverse problem with spatial regularization to a neighboring image. Technically, this task is also accomplished by the Gauss-Newton method. Finally, during post-processing the resulting images are denoised with a modified non-local means filter (15). In addition, the non-DW and DW images [and therefore also the calculated apparent diffusion coefficient (ADC) maps] are masked with an absolute intensity threshold. It may be selected by the radiologic user and in this study corresponds to about $1.5 \%$ of the mean intensity of the non-DW images. For each cross-sectional slice the standard output for DW singleshot STEAM MRI comprises a (mean) non-DW image (in case of multiple averages), a mean DW image averaged across directions (and repetitions if any), and a map of the ADC. For only two b-values and 6 directions the latter may directly be obtained from the trace-weighted images according to $\mathrm{ADC}=[\ln \operatorname{Tr}(\mathrm{b} 1)-\ln \operatorname{Tr}(\mathrm{b} 2)] /(\mathrm{b} 1-\mathrm{b} 2)$.

All reconstructions were performed online and rapidly completed after the end of data acquisition. For this purpose, the algorithm was implemented on a computer with multiple graphics processing units GeForce GTX 

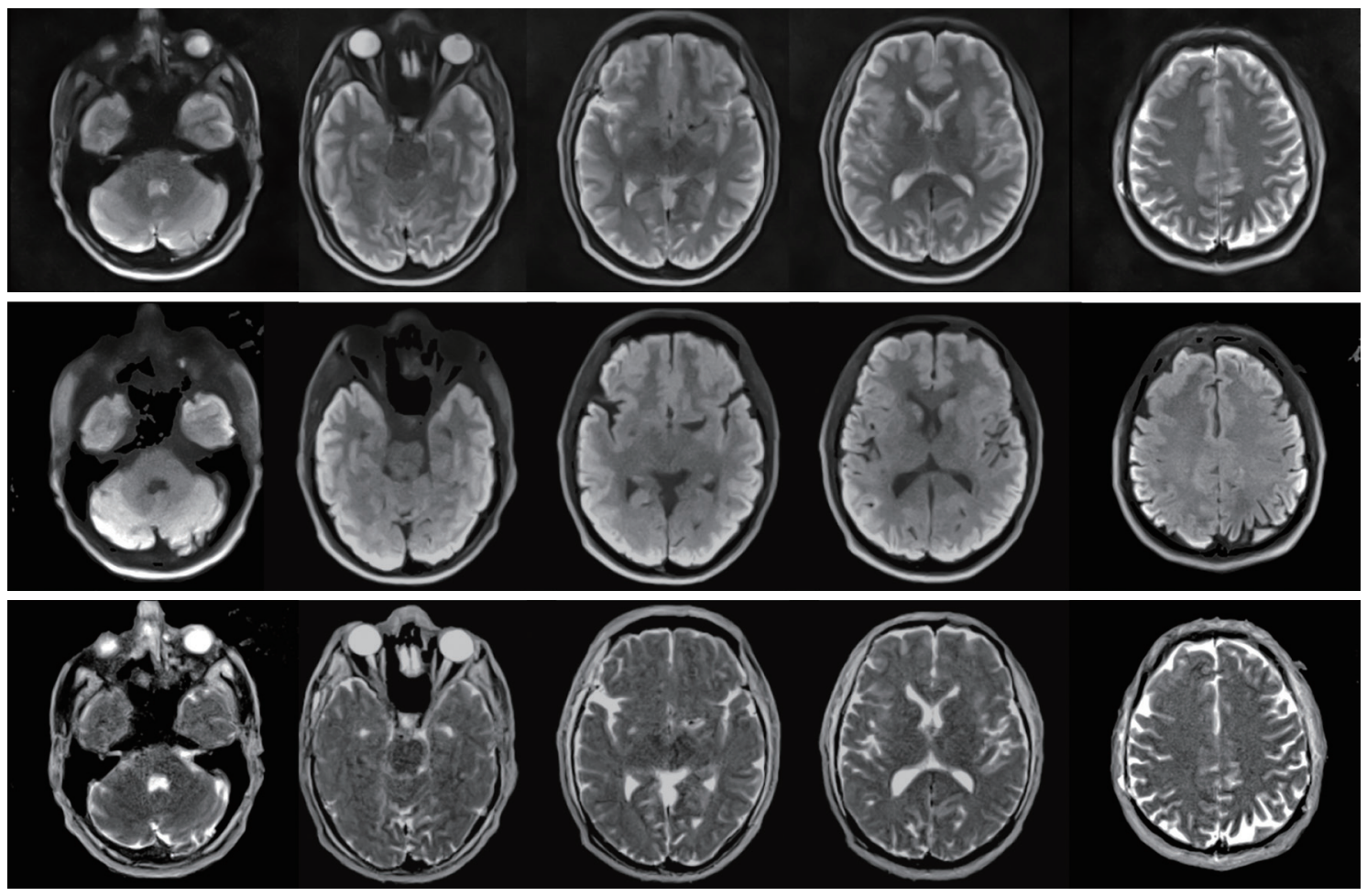

Figure 2 (Top row) non-diffusion-weighted (non-DW) images $(\mathrm{b}=0)$, (middle) mean DW images $\left(\mathrm{b}=1,000 \mathrm{~s} / \mathrm{mm}^{2}, 6\right.$ gradient directions) and (bottom) apparent diffusion coefficient (ADC) maps of the brain of a healthy volunteer as obtained by DW single-shot stimulated echo acquisition mode (STEAM) MRI. The 5 slices (left to right) are selected from a series of 51 directly neighboring cross-sections at 3-mm thickness. The total measuring time was $2 \mathrm{~min} 30 \mathrm{~s}$. For other details see Table 1 .

TITAN (NVIDIA, Santa Clara, CA, USA). It is connected by a high-speed link to the host computer of the MRI system and operates invisible to the user.

A preliminary validation of the technical performance and achievable image quality of DW single-shot STEAM MRI was obtained by proof-of-principle applications to the brain and prostate of healthy subjects. For this purpose, simple "clinical" protocols were chosen with two b values and diffusion-encoding gradients along 6 directions. Experimental parameters for brain and prostate studies are summarized in Table 1.

\section{Results}

An example for DW single-shot STEAM MRI of the normal brain is shown in Figure 2. It presents 5 selected slices (left to right) of a whole-brain study where non-DW and DW images ( $\mathrm{b}=1,000 \mathrm{~s} \mathrm{~mm}^{-2}, 6$ diffusion directions) were acquired for 51 directly neighboring cross-sections of $3-\mathrm{mm}$ thickness in a total measurement time of $2 \mathrm{~min}$ and 30 s. The results in Figure 2 refer to (top row) the nonDW images, (middle) the mean DW images and (bottom) the ADC maps. Regardless of whether the images are from transverse brain sections with (right) little or (left) strong natural differences in magnetic susceptibility, all images are free from respective artefacts, i.e., geometric distortions and signal alterations. Moreover, for acquisitions with three non-DW images and two DW images per gradient direction (see Table 1), the resulting diffusion maps promise diagnostic quality within a clinically feasible measuring time. If even faster protocols are required and a somewhat reduced SNR is acceptable, i.e., without any averaging, the minimum scan time for whole-brain DW single-shot STEAM MRI reduces to $1 \mathrm{~min}$ and $10 \mathrm{~s}$ for 6 gradient directions or even $40 \mathrm{~s}$ when using only three directions. For the subject shown in Figure 2 preliminary evaluations revealed mean $\mathrm{ADC}$ values (multiple regions) for cortical gray and white matter of $(1.04 \pm 0.04) \times 10^{-3}$ and $(0.75 \pm 0.05)$ $\times 10^{-3} \mathrm{~mm}^{2} / \mathrm{s}$, respectively.

The observation of high-quality images without 

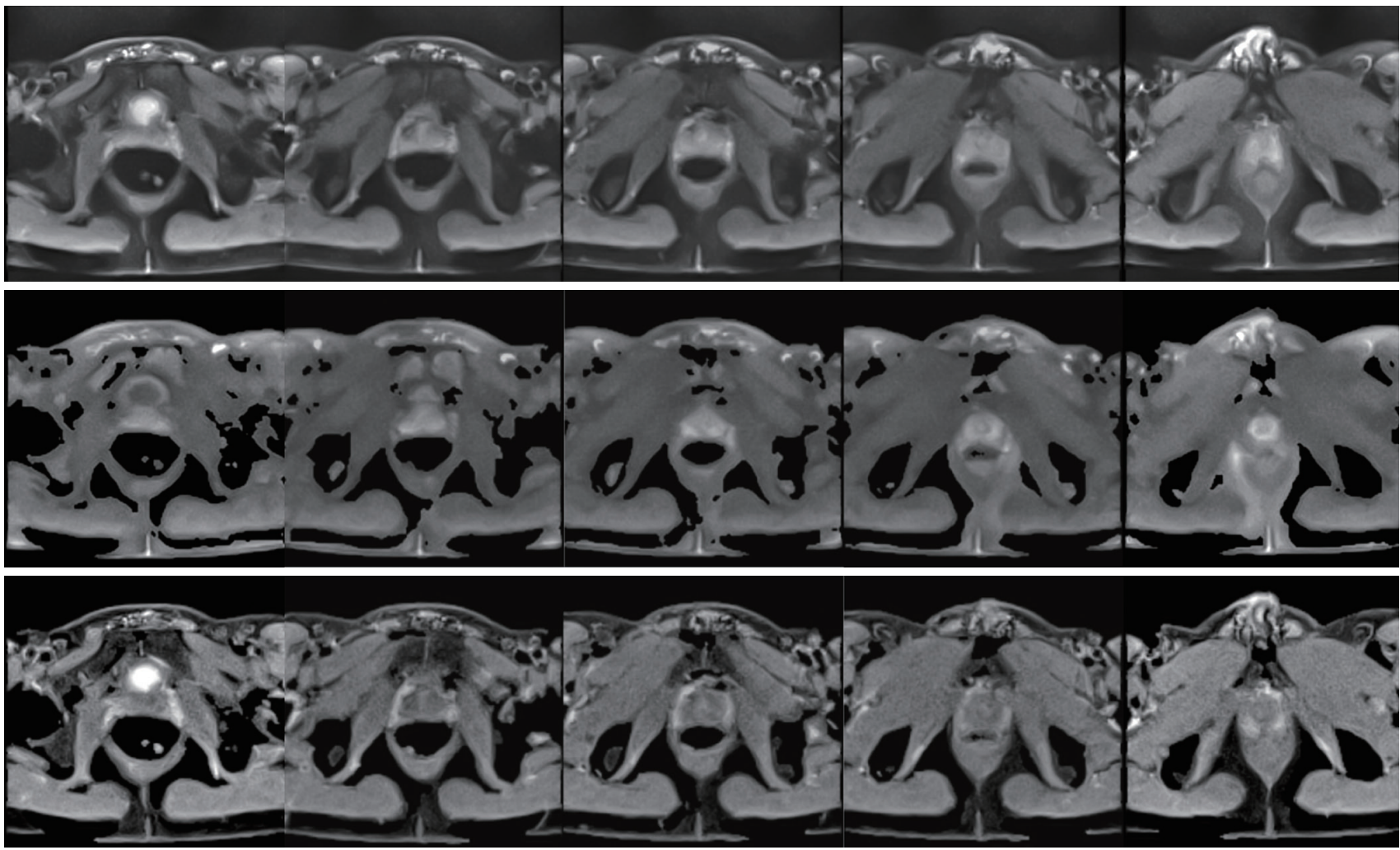

Figure 3 (Top row) non-diffusion-weighted (non-DW) images $(\mathrm{b}=0)$, (middle) mean DW images $\left(\mathrm{b}=600 \mathrm{~s} / \mathrm{mm}^{2}, 6\right.$ gradient directions) and (bottom) apparent diffusion coefficient (ADC) maps of the prostate of a healthy volunteer as obtained by DW single-shot stimulated echo acquisition mode (STEAM) MRI. The 5 slices (left to right, every other section) are selected from a series of 31 directly neighboring crosssections at 3-mm thickness. The total measuring time was 4 min 30 s. For other details see Table 1.

susceptibility artifacts also holds true for a DW singleshot STEAM MRI study of the normal human prostate shown in Figure 3. This is most remarkable because of the close vicinity of the air-filled rectum. Moreover, these measurements were performed without the use of an endorectal coil and during free breathing. Similar to the data shown in Figure 2, (top row) the non-DW images, (middle) the mean DW images $\left(\mathrm{b}=600 \mathrm{~s} / \mathrm{mm}^{2}, 6\right.$ gradient directions), and (bottom) the ADC maps of 5 cross-sections (every other slice centered on the prostate) were selected from a total of 31 directly neighboring slices of $3-\mathrm{mm}$ thickness. The measurement time was $4 \mathrm{~min}$ and $30 \mathrm{~s}$ when averaging 9 non-DW images and $6 \mathrm{DW}$ images per direction (see Table 1). The mean ADC value for the central gland (multiple sections) yielded $(1.27 \pm 0.11) \times 10^{-3} \mathrm{~mm}^{2} / \mathrm{s}$

\section{Discussion}

This work reports the development of a novel DW single- shot STEAM MRI technique which overcomes previous limitations by extending the inverse reconstruction by spatial regularization. The method covers a volume by a series of directly neighboring slices and successfully exploits the spatial similarity of neighboring images. Preliminary applications to the normal brain and prostate demonstrate (I) the absence of geometric distortions and false signal alterations, (II) a high degree of data undersampling and correspondingly short acquisition times, (III) computational stability, spatial fidelity and online reconstruction, (IV) adequate SNR and spatial resolution, and (V) clinically feasible scan times.

Another advantage of the method is its applicability to different organ systems, in particular to both the human brain and prostate, without the need for technical or mathematical adjustments-apart from the choice of suitable experimental parameters such as spatial resolution or number of averages. This is in contrast to preceding DW STEAM MRI versions with insufficient regularization 
which were exclusively designed for studies of the brain (9) or prostate (16).

A limitation of this brief report about a technical development is the use of only very simple diffusion protocols with two $\mathrm{b}$ values and 6 directions. This is because the primary aim was the evaluation of a new method with emphasis on technical feasibility and achievable image quality. Moreover, the present work does not offer any quantitative comparisons, for example to DW EPI. On the other hand, there is previous information about the different performance of STEAM and EPI, e.g., see $(8,16)$, while clinically meaningful comparisons are beyond the scope of this work and will be the subject of forthcoming studies. A final limitation is the focus on healthy volunteers which nevertheless serves the qualitative purpose outlined above. It is quite clear that having demonstrated clinically relevant benefits, the next step must be an assessment of the diagnostic potential of DW single-shot STEAM MRI in extended patient studies.

\section{Acknowledgments}

Funding: None.

\section{Footnote}

Conflicts of Interest: All authors have completed the ICMJE uniform disclosure form (available at http://dx.doi. org/10.21037/qims-20-871). The authors report that they are co-inventors of a pending patent "Diffusion-weighted MRI of a volume without susceptibility artifacts".

Ethical Statement: The study was approved by the ethics committee of the Göttingen University Medicine (No. $12 / 6 / 15)$ and written informed consent was taken from all subjects prior to MRI.

Open Access Statement: This is an Open Access article distributed in accordance with the Creative Commons Attribution-NonCommercial-NoDerivs 4.0 International License (CC BY-NC-ND 4.0), which permits the noncommercial replication and distribution of the article with the strict proviso that no changes or edits are made and the original work is properly cited (including links to both the formal publication through the relevant DOI and the license). See: https://creativecommons.org/licenses/by-nc-nd/4.0/.

\section{References}

1. Chilla GS, Tan CH, Xu C, Poh CL. Diffusion weighted magnetic resonance imaging and its recent trend-a survey. Quant Imaging Med Surg 2015;5:407-22.

2. Baliyan V, Das CJ, Sharma R, Gupta AK. Diffusion weighted imaging: Technique and applications. World J Radiol 2016;8:785-98.

3. Schouten CS, de Bree R, van der Putten L, Noij DP, Hoekstra OS, Comans EF, Witte BI, Doornaert PA, Leemans CR, Castelijns JA. Diffusion-weighted EPIand HASTE-MRI and 18F-FDG-PET-CT early during chemoradiotherapy in advanced head and neck cancer. Quant Imaging Med Surg 2014;4:239-50.

4. Hirata K, Nakaura T, Okuaki T, Kidoh M, Oda S, Utsunomiya D, Namimoto T, Kitajima M, Nakayama H, Yamashita Y. Comparison of the image quality of turbo spin echo- and echo-planar diffusion-weighted images of the oral cavity. Medicine (Baltimore) 2018;97:e0447.

5. Frahm J, Haase A, Matthaei D, Merboldt KD, Hänicke. Rapid NMR imaging using stimulated echoes. J Magn Reson 1985;65:130-5.

6. Merboldt KD, Hänicke W, Bruhn H, Gyngell ML, Frahm J. Diffusion imaging of the human brain in vivo using highspeed STEAM MRI. Magn Reson Med 1992;23:179-92.

7. Nolte UG, Finsterbusch J, Frahm J. Rapid isotropic diffusion mapping without susceptibility artifacts: whole brain studies using diffusion-weighted single-shot STEAM MR imaging. Magn Reson Med 2000;44:731-6.

8. Khalil AA, Hohenhaus M, Kunze C, Schmidt W, Brunecker P, Villringer K, Merboldt KD, Frahm J, Fiebach JB. Sensitivity of Diffusion-Weighted STEAM MRI and EPI-DWI to Infratentorial Ischemic Stroke. PLoS One 2016;11:e0161416.

9. Merrem A, Hofer S, Voit D, Merboldt KD, Klosowski J, Untenberger M, Fleischhammer J, Frahm J. Rapid Diffusion-Weighted Magnetic Resonance Imaging of the Brain Without Susceptibility Artifacts: Single-Shot STEAM With Radial Undersampling and Iterative Reconstruction. Invest Radiol 2017;52:428-33.

10. Uecker M, Zhang S, Voit D, Karaus A, Merboldt KD, Frahm J. Real-time MRI at a resolution of $20 \mathrm{~ms}$. NMR Biomed 2010;23:986-94.

11. Voit D, Kalentev O, van Zalk M, Joseph AA, Frahm J. Rapid and motion-robust volume coverage using cross-sectional real-time MRI. Magn Reson Med 2020;83:1652-8.

12. Frahm J, Schätz S, Untenberger M, Zhang S, Voit D, 
Merboldt KD, Sohns JM, Lotz J, Uecker M. On the Temporal Fidelity of Nonlinear Inverse Reconstructions for Real-Time MRI - The Motion Challenge. Open Med Imaging J 2014;8:1-7.

13. Haase A, Frahm J, Hänicke W, Matthaei D. 1H NMR chemical shift selective (CHESS) imaging. Phys Med Biol 1985;30:341-4.

14. Bakushinsky AB, Kokurin MY. Iterative Methods for Approximate Solution of Inverse Problems. Dordrecht,

Cite this article as: Voit D, Kalentev O, Frahm J. Diffusionweighted magnetic resonance imaging (MRI) without susceptibility artifacts: single-shot stimulated echo acquisition mode (STEAM) MRI with iterative reconstruction and spatial regularization. Quant Imaging Med Surg 2021;11(2):831-837. doi: 10.21037/qims-20-871
The Netherlands: Springer, 2005.

15. Klosowski J, Frahm J. Image denoising for real-time MRI. Magn Reson Med 2017;77:1340-52.

16. Merrem A, Hofer S, Seif Amir Hosseini A, Voit D, Merboldt KD, Tan Z, Frahm J. Diffusion-weighted MRI of the prostate without susceptibility artifacts: Undersampled multi-shot turbo-STEAM with rotated radial trajectories. NMR Biomed 2019;32:e4074. 